\title{
Supernova Nucleosynthesis in Massive Stars
}

\author{
By M. HASHIMOTO, ${ }^{1}$ K. NOMOTO ${ }^{2}$ T. TSUJIMOTO, \\ AND F. - K. THIELE M A N N \\ ${ }^{1}$ Kyushu University, Fukuoka 810, Japan \\ ${ }^{2}$ University of Tokyo, Bunkyo-ku, Tokyo 113, Japan \\ ${ }^{3}$ Harvard-Smithsonian Center for Astrophysics, 60 Garden St., Cambridge, MA 02138, USA
}

Presupernova evolution and explosive nucleosynthesis in massive stars for main-sequence masses from $13 M_{\odot}$ to $70 M_{\odot}$ are calculated. We examine the dependence of the supernova yields on the stellar mass, ${ }^{12} \mathrm{C}(\alpha, \gamma){ }^{16} \mathrm{O}$ rate, and explosion energy. The supernova yields integrated over the initial mass function are compared with the solar abundances.

\section{Presupernova models and the ${ }^{12} \mathrm{C}(\alpha, \gamma)^{16} \mathrm{O}$ rate}

Presupernova models are obtained for helium stars with masses of $M_{\alpha}=3.3,4,5$, $6,8,16$, and $32 M_{\odot}$ as an extension of the studies by Nomoto \& Hashimoto (1988), Thielemann et al. (1993), and Hashimoto et al. (1993). These helium star masses correspond approximately to main-sequence masses of $M_{\mathrm{ms}}=13,15,18,20,25,40$, and $70 M_{\odot}$, respectively (Sugimoto \& Nomoto 1980). The systematic study for such a dense grid of stellar masses enables us to understand how explosive nucleosynthesis depends on the presupernova stellar structure and to apply the results to the chemical evolution of galaxies. We use the Schwarzschild criterion for convection and neglect overshooting. The initial composition is given by $X\left({ }^{4} \mathrm{He}\right)=0.9879$ and $X\left({ }^{14} \mathrm{~N}\right)=0.0121$. These helium stars are evolved from helium burning through the onset of the Fe core collapse.

Nuclear reaction rates are mostly taken from Caughlan \& Fowler (1988). For the uncertain rate of ${ }^{12} \mathrm{C}(\alpha, \gamma){ }^{16} \mathrm{O}$, we use the rate by Caughlan et al. (1985; CFHZ85), which is larger than the rate by Caughlan \& Fowler (1988; CF88) by a factor of $\sim 2.4$. To examine the influence of this difference, we evolve the $M_{\alpha}=8 M_{\odot}$ helium star, using the ${ }^{12} \mathrm{C}(\alpha, \gamma){ }^{16} \mathrm{O}$ rate by CF88 (case 25B). [The $25 M_{\odot}$ star model with the ${ }^{12} \mathrm{C}(\alpha, \gamma){ }^{16} \mathrm{O}$ rate by CFHZ85 is denoted as case 25A.] At the end of core helium burning, the formation of the carbon-oxygen core and its composition are influenced largely by the ${ }^{12} \mathrm{C}(\alpha, \gamma){ }^{16} \mathrm{O}$ rate. The larger rate results in a smaller $\mathrm{C} / \mathrm{O}$ ratio, which affects the abundances of $\mathrm{Ne}$, $\mathrm{Mg}, \mathrm{Al}$ relative to $\mathrm{O}$ in the more evolved cores.

Comparison of the presupernova density structures for the two cases $25 \mathrm{~A}$ and $25 \mathrm{~B}$ shows that case $25 \mathrm{~B}$ has a more concentrated core at $M_{r}<2 M_{\odot}$ (i.e., a steeper density gradient) and more extended outer layers than case $25 \mathrm{~A}$. This is due to a larger carbon abundance and thus stronger carbon shell burning in case $25 \mathrm{~B}$.

It is found that the size of the iron core is not a monotonic function of the helium core mass as shown by Barkat \& Marom (1990) and Woosley (1993). For $M_{\mathrm{ms}}=13,15,18$, 20,25 (case $25 \mathrm{~A}$ ) , 40, and $70 M_{\odot}$, the iron core masses are $1.18,1.28,1.36,1.40,1.42$, 1.88 , and $1.57 M_{\odot}$, respectively. In case $25 \mathrm{~B}$, the iron core mass is $1.37 M_{\odot}$, which is smaller than in case $25 \mathrm{~A}$. 


\section{Explosive nucleosynthesis}

The hydrodynamic phases of supernova explosions for the above eight presupernova models were followed with an extensive nuclear reaction network (Hashimoto et al. 1989, 1993; Thielemann et al. 1990, 1993).

Since the mechanism of supernova explosions after core collapse is not fully understood yet, the explosion energy and the mass cut (or ${ }^{56} \mathrm{Ni}$ mass) have remaining uncertainties, except for SN 1987A. The final kinetic energy of the explosion is assumed to be $E=1.0$ $\times 10^{51}$ erg as inferred from the modeling of SN 1987A and SN 1993J (e.g., Shigeyama \& Nomoto 1990; Shigeyama et al. 1994).

In the present study, the mass cut is chosen to produce $0.075 M_{\odot}{ }^{56} \mathrm{Ni}$ for $13-70 M_{\odot}$ stars. This is based on the estimates from the light curves of SN 1993J for the $13-15$ $M_{\odot}$ stars (e.g., Nomoto et al. 1993; Wheeler \& Filippenko 1993) and SN 1987A for the $18-20 M_{\odot}$ stars (e.g., Nomoto et al. 1993). For more massive stars, a similar mass of ${ }^{56} \mathrm{Ni}$ is suggested from SN $1990 \mathrm{E}$ (Schmidt et al. 1993) and also from the absence of very bright type Ib supernovae with slow decline (e.g., Shigeyama et al. 1990).

Figures 1 and 2 show the integrated abundances of the ejecta relative to the solar values (Anders \& Grevesse 1989) for $M_{\mathrm{ms}}=13,15,18,20,40$, and $70 M_{\odot}$, respectively. Figure 3 shows three cases of $M_{\mathrm{ms}}=25 M_{\odot}$, i.e., cases $25 \mathrm{~A}, 25 \mathrm{~B}$, and $25 \mathrm{BE}$ (see below). 

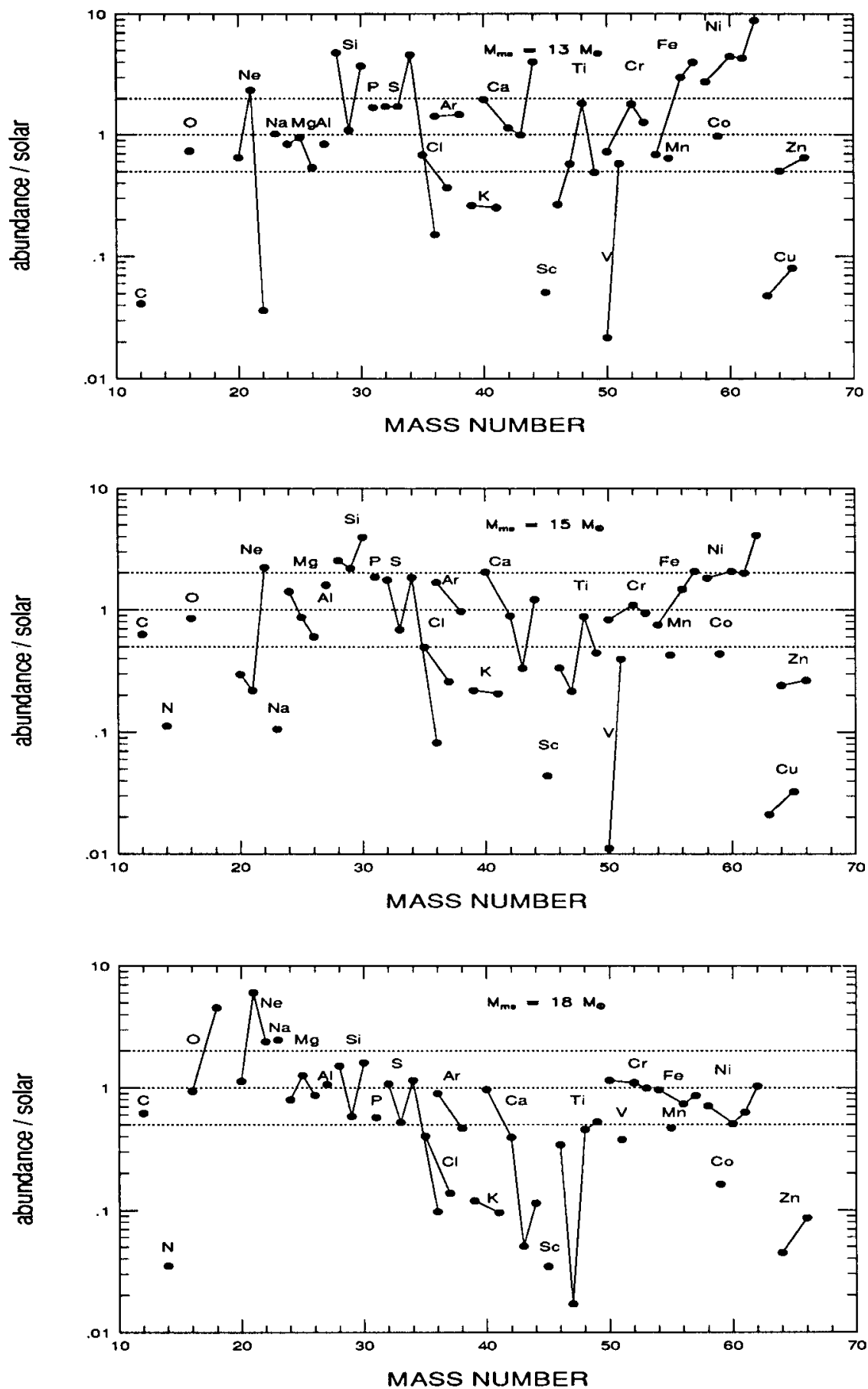

Figure 1. Abundances of stable isotopes relative to the solar values for the 13,15 , and $18 M_{\odot}$ stars. 

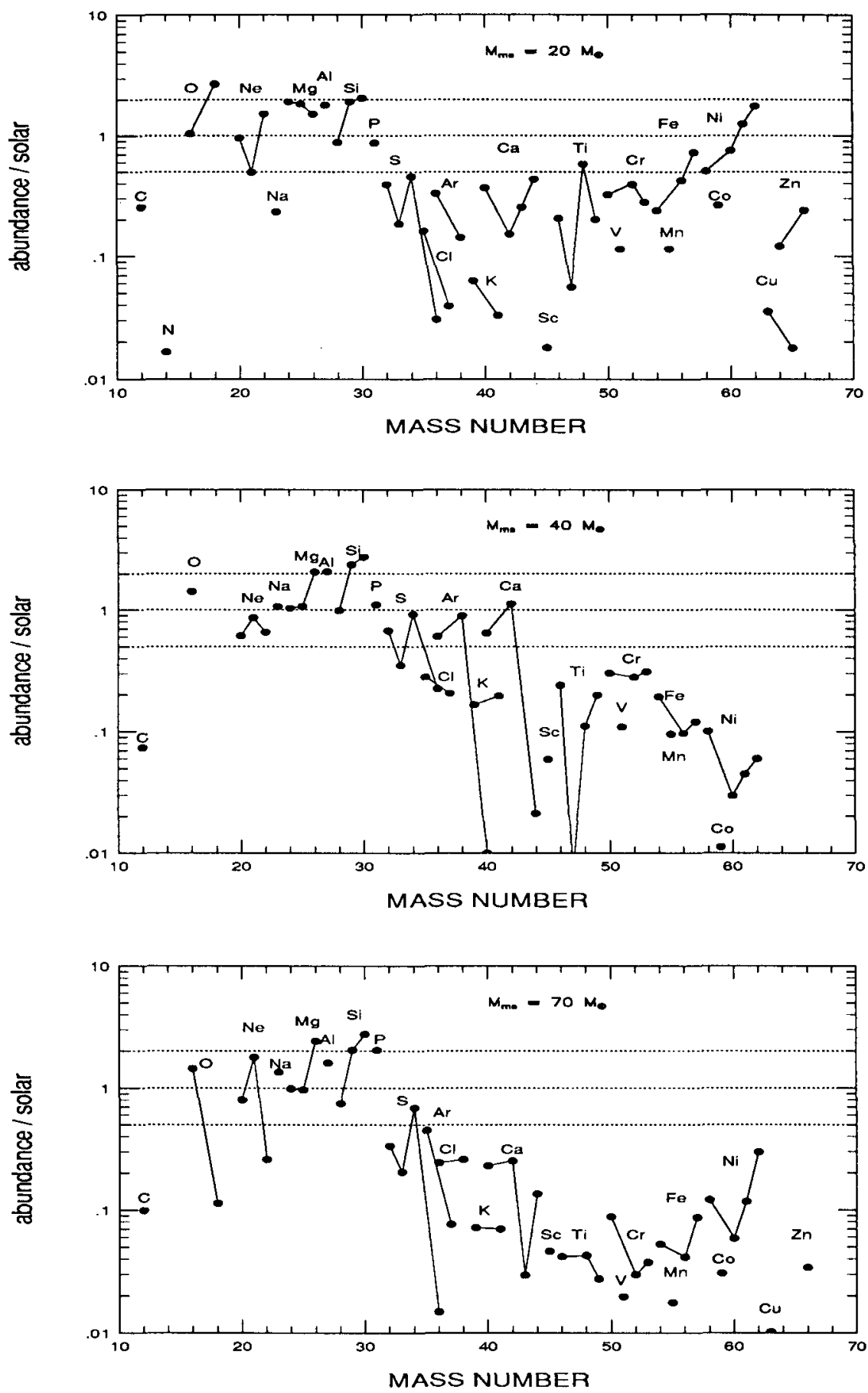

FIgure 2. Same as Fig. 1 but for the 20,40 , and $70 M_{\odot}$ stars. 

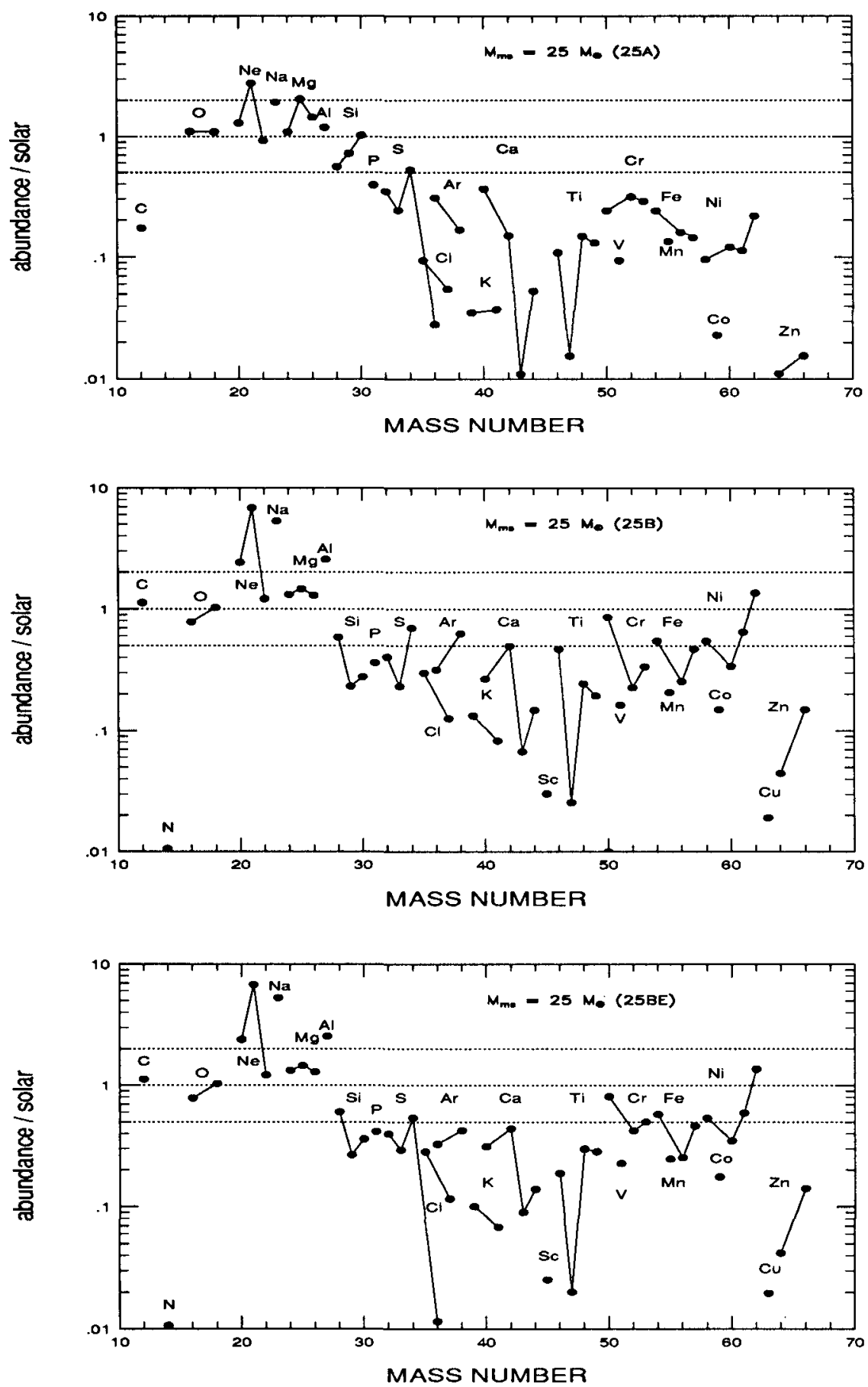

Figure 3. Same as in Fig. 1 but for the $25 M_{\odot}$ star (case 25A). Case 25B uses the ${ }^{12} \mathrm{C}(\alpha, \gamma){ }^{16} \mathrm{O}$ rate by $\mathrm{CF} 88$ and case $25 \mathrm{BE}$ is the same as $25 \mathrm{~B}$ but with $E=1.5 \times 10^{51} \mathrm{erg} \mathrm{s}^{-1}$. 


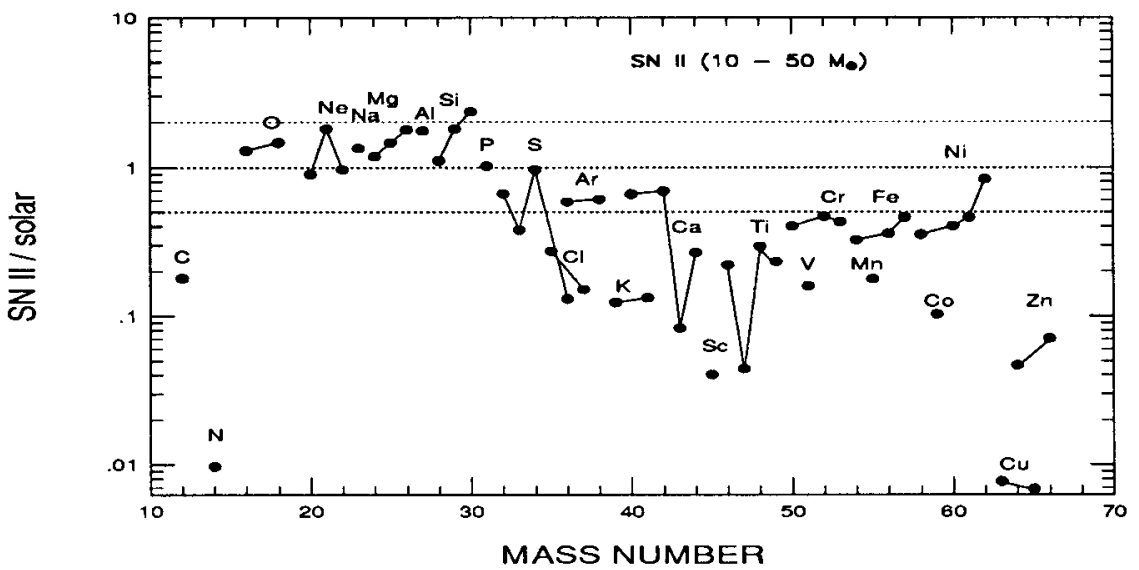

Figure 4. Nucleosynthesis products from $10-50 M_{\odot}$ stars averaged over the IF relative to solar abundances.

TABLE 1. Nucleosynthesis products (in $M_{\odot}$ ) from $10-50 M_{\odot}$ stars averaged over the IMF.

\begin{tabular}{rccccc}
\hline${ }^{12} \mathrm{C}$ & $7.93 \mathrm{E}-02$ & ${ }^{35} \mathrm{Cl}$ & $1.01 \mathrm{E}-04$ & ${ }^{54} \mathrm{Cr}$ & $1.83 \mathrm{E}-08$ \\
${ }^{13} \mathrm{C}$ & $3.80 \mathrm{E}-09$ & ${ }^{37} \mathrm{Cl}$ & $1.88 \mathrm{E}-05$ & ${ }^{55} \mathrm{Mn}$ & $3.44 \mathrm{E}-04$ \\
${ }^{14} \mathrm{~N}$ & $1.56 \mathrm{E}-03$ & ${ }^{36} \mathrm{Ar}$ & $6.62 \mathrm{E}-03$ & ${ }^{54} \mathrm{Fe}$ & $3.38 \mathrm{E}-03$ \\
${ }^{15} \mathrm{~N}$ & $1.66 \mathrm{E}-08$ & ${ }^{38} \mathrm{Ar}$ & $1.37 \mathrm{E}-03$ & ${ }^{56} \mathrm{Fe}$ & $6.10 \mathrm{E}-02$ \\
${ }^{16} \mathrm{O}$ & 1.80 & ${ }^{40} \mathrm{Ar}$ & $2.27 \mathrm{E}-08$ & ${ }^{57} \mathrm{Fe}$ & $1.92 \mathrm{E}-03$ \\
${ }^{17} \mathrm{O}$ & $9.88 \mathrm{E}-08$ & ${ }^{39} \mathrm{~K}$ & $6.23 \mathrm{E}-05$ & ${ }^{58} \mathrm{Fe}$ & $6.43 \mathrm{E}-09$ \\
${ }^{18} \mathrm{O}$ & $4.61 \mathrm{E}-03$ & ${ }^{41} \mathrm{~K}$ & $5.07 \mathrm{E}-06$ & ${ }^{59} \mathrm{Co}$ & $5.01 \mathrm{E}-05$ \\
${ }^{19} \mathrm{~F}$ & $1.16 \mathrm{E}-09$ & ${ }^{40} \mathrm{Ca}$ & $5.77 \mathrm{E}-03$ & ${ }^{58} \mathrm{Ni}$ & $2.54 \mathrm{E}-03$ \\
${ }^{20} \mathrm{Ne}$ & $2.12 \mathrm{E}-01$ & ${ }^{42} \mathrm{Ca}$ & $4.23 \mathrm{E}-05$ & ${ }^{60} \mathrm{Ni}$ & $1.15 \mathrm{E}-03$ \\
${ }^{21} \mathrm{Ne}$ & $1.08 \mathrm{E}-03$ & ${ }^{43} \mathrm{Ca}$ & $1.08 \mathrm{E}-06$ & ${ }^{61} \mathrm{Ni}$ & $5.76 \mathrm{E}-05$ \\
${ }^{22} \mathrm{Ne}$ & $1.83 \mathrm{E}-02$ & ${ }^{44} \mathrm{Ca}$ & $5.53 \mathrm{E}-05$ & ${ }^{62} \mathrm{Ni}$ & $3.37 \mathrm{E}-04$ \\
${ }^{23} \mathrm{Na}$ & $6.51 \mathrm{E}-03$ & ${ }^{46} \mathrm{Ca}$ & $1.43 \mathrm{E}-10$ & ${ }^{64} \mathrm{Ni}$ & $3.18 \mathrm{E}-14$ \\
${ }^{24} \mathrm{Mg}$ & $8.83 \mathrm{E}-02$ & ${ }^{48} \mathrm{Ca}$ & $5.33 \mathrm{E}-14$ & ${ }^{63} \mathrm{Cu}$ & $6.45 \mathrm{E}-07$ \\
${ }^{25} \mathrm{Mg}$ & $1.44 \mathrm{E}-02$ & ${ }^{45} \mathrm{Sc}$ & $2.29 \mathrm{E}-07$ & ${ }^{65} \mathrm{Cu}$ & $2.65 \mathrm{E}-07$ \\
${ }^{26} \mathrm{Mg}$ & $2.01 \mathrm{E}-02$ & ${ }^{46} \mathrm{Ti}$ & $7.19 \mathrm{E}-06$ & ${ }^{64} \mathrm{Zn}$ & $6.76 \mathrm{E}-06$ \\
${ }^{27} \mathrm{Al}$ & $1.48 \mathrm{E}-02$ & ${ }^{47} \mathrm{Ti}$ & $1.34 \mathrm{E}-06$ & ${ }^{66} \mathrm{Zn}$ & $6.06 \mathrm{E}-06$ \\
${ }^{28} \mathrm{Si}$ & $1.05 \mathrm{E}-01$ & ${ }^{48} \mathrm{Ti}$ & $9.23 \mathrm{E}-05$ & ${ }^{67} \mathrm{Zn}$ & $1.19 \mathrm{E}-08$ \\
${ }^{29} \mathrm{Si}$ & $8.99 \mathrm{E}-03$ & ${ }^{49} \mathrm{Ti}$ & $5.55 \mathrm{E}-06$ & ${ }^{68} \mathrm{Zn}$ & $2.73 \mathrm{E}-09$ \\
${ }^{30} \mathrm{Si}$ & $8.05 \mathrm{E}-03$ & ${ }^{50} \mathrm{Ti}$ & $2.95 \mathrm{E}-10$ & ${ }^{69} \mathrm{Ga}$ & $2.60 \mathrm{E}-12$ \\
${ }^{31} \mathrm{P}$ & $1.21 \mathrm{E}-03$ & ${ }^{50} \mathrm{~V}$ & $6.19 \mathrm{E}-10$ & ${ }^{71} \mathrm{Ga}$ & $1.37 \mathrm{E}-14$ \\
${ }^{32} \mathrm{~S}$ & $3.84 \mathrm{E}-02$ & ${ }^{51} \mathrm{~V}$ & $8.73 \mathrm{E}-06$ & ${ }^{70} \mathrm{Ge}$ & $1.65 \mathrm{E}-12$ \\
${ }^{33} \mathrm{~S}$ & $1.78 \mathrm{E}-04$ & ${ }^{50} \mathrm{Cr}$ & $4.34 \mathrm{E}-05$ & ${ }^{72} \mathrm{Ge}$ & $1.61 \mathrm{E}-12$ \\
${ }^{34} \mathrm{~S}$ & $2.62 \mathrm{E}-03$ & ${ }^{52} \mathrm{Cr}$ & $1.01 \mathrm{E}-03$ & ${ }^{73} \mathrm{Ge}$ & $9.25 \mathrm{E}-17$ \\
${ }^{36} \mathrm{~S}$ & $1.78 \mathrm{E}-06$ & ${ }^{53} \mathrm{Cr}$ & $1.08 \mathrm{E}-04$ & & \\
\hline
\end{tabular}

To examine the dependence on the explosion energy, we show the case $25 \mathrm{BE}$, i.e., case $25 \mathrm{~B}$ with $E=1.5 \times 10^{51} \mathrm{erg}$. The larger explosion energy leads to the outward shift of the abundance distribution. This leads to minor differences between the abundances for the two explosion energies (Fig. 3). 


\section{Isotopic abundance ratios}

Figure 4 shows the isotopic abundances relative to their solar values (Anders \& Grevesse 1989) after averaging over the mass range from 10 to $50 M_{\odot}$ with an initial mass function $\propto M^{-1.35}$. Here the upper mass limit $50 M_{\odot}$ is chosen from the comparison of $[\mathrm{O} / \mathrm{Fe}]$ and $[\mathrm{Mg} / \mathrm{Fe}]$ with those of metal-poor stars (Tsujimoto et al. 1994). We also assume no heavy element production below $10 M_{\odot}$ and approximate the abundances of $10-13$ $M_{\odot}$ stars by a linear interpolation between 10 and $13 M_{\odot}$. Table 1 gives the integrated masses $\left(M_{\odot}\right)$ of stable species averaged over the initial mass function.

Figure 4 shows that the relative abundance ratios from massive stars are in good agreement with the solar ratios for $A<27$. [The sum of type Ia and type II products with a ratio of 1 to 9 reproduces well the solar abundances for a wider range of $A$ (Tsujimoto et al. 1993).] Note that this agreement is realized for the ${ }^{12} \mathrm{C}(\alpha, \gamma){ }^{16} \mathrm{O}$ rate by CFHZ85, i.e., case 25A. For case 25B, $\mathrm{Ne}, \mathrm{Na}$, and $\mathrm{Al}$ relative to $\mathrm{O}$ are overproduced with respect to the solar ratios as seen in Figure 3. This is due to the larger $\mathrm{C} / \mathrm{O}$ ratio in case $25 \mathrm{~B}$ after helium burning. Since the products of the $25 M_{\odot}$ star dominate type II supernova yields, this result suggests that the ${ }^{12} \mathrm{C}(\alpha, \gamma){ }^{16} \mathrm{O}$ rate is higher than that of CF88 and closer to CFHZ85. The presently most reliable experimental investigations give values in between the two rates.

We should note that the isotopic ratios in Figures $1-4$ depend not only on the ${ }^{12} \mathrm{C}(\alpha, \gamma){ }^{16} \mathrm{O}$ rate but also on convective overshooting, mixing fresh He into the core at late high temperature core helium burning stages. The above comparison that favors the CFHZ85 rate is based on the calculations with no convective overshooting. If overshooting during convective core helium burning would reduce the $\mathrm{C} / \mathrm{O}$ ratio, a smaller ${ }^{12} \mathrm{C}(\alpha, \gamma){ }^{16} \mathrm{O}$ rate would be favored (Weaver \& Woosley 1993).

Figure 4 also shows that some species, ${ }^{35} \mathrm{Cl},{ }^{39} \mathrm{~K},{ }^{44} \mathrm{Ca},{ }^{48} \mathrm{Ti}$, and ${ }^{59} \mathrm{Co}$, are underproduced relative to the solar values. If we include the weak component of the s-process nuclei $50<A<100$ produced during core helium burning (Prantzos et al. 1990 ), ${ }^{48} \mathrm{Ti}$ and ${ }^{59} \mathrm{Co}$ are enhanced appreciably compared with the seed (solar) abundances. ${ }^{35} \mathrm{Cl}$, ${ }^{39} \mathrm{~K}$ and ${ }^{44} \mathrm{Ca}$ are enhanced only by a factor of $\sim 2$. Synthesis of s-process elements during carbon shell burning would also be significant (Raiteri et al. 1993).

\section{Acknowledgements}

This work has been supported in part by the Grant in Aid for Scientific Research $(04640265,05242102,05242103,05242207,05243206,3883)$ of the Ministry of Education, Science, and Culture in Japan and NSF-grant AST 89-13799.

\section{REFERENCES}

Anders, E., \& Grevesse, N. 1989, Geochim. Cosmochim. Acta, 53, 197

Barkat, Z., \& Marom, A. 1990, in Supernovae, ed. J.C. Wheeler et al. (World Scientific), p. 95

Caughlan, G. R., Fowler, W. A., Harris, M. J., \& Zimmerman, B.A. 1985, Atomic Data \& Nuclear Data Tables, 32, 197

Caughlan, G. R., \& Fowler, W. A. 1988, Atomic Data \& Nucl. Data Tables, 40, 283

Hashimoto, M., Nomoto, K., \& Shigeyama, T. 1989, A\&A 210, L5

Hashimoto, M., Nomoto, K., Tsujimoto, T., \& Thielemann, F.-K. 1993, in Nuclei in Cosmos, ed. F. Käppeler \& K. Wisshak (Institute Physics Publ.), p. 587

Nomoto, K., \& Hashimoto, M. 1988, Phys. Rep., 163, 13

Nomoto, K., Shigeyama, T., Kumagai, S., Yamaoka, H., \& Suzuki, T. 1993, in Supernovae (Les Houches, Session LIV), ed. S. Bludman, R. et al. (Elsevier Sci. Publ.) 
Nomoto, K., Suzuki, T., Shigeyama, T., Kumagai, S., Yamaoka, H., \& Saio, H. 1993, Nature, 364,507

Prantzos, N., Hashimoto, M., \& Nomoto, K. 1990, A\&A, 234, 211

Raiteri, C. M., Gallino, R., Busso, M., Neuberger, D., \& Käppeler F. 1993, ApJ, 419, 207

Schmidt, B. P. et al. 1993, AJ, 105, 2236

Shigeyama, T., \& Nomoto, K. 1990, ApJ, 360, 242

Shigeyama, T., Nomoto, K., Tsujimoto, T. \& Hashimoto, M. 1990, ApJ, 361, L23

Shigeyama, T., Suzuki, T., Kumagai, S., Nomoto, K., Saio, H., \& Yamaoka, H. 1994, ApJ, 420, 341

Sugimoto, D., \& Nomoto, K. 1980, Space Sci. Rev. 25, 155

Thielemann, F.-K., Hashimoto, M., \& Nomoto, K. 1990, ApJ, 349, 222

Thielemann, F.-K., Nomoto, K., \& Hashimoto, M. 1993, in Les Houches, Session LIV ed. S. Bludman et al. (Elsevier Sci. Publ.)

Tsujimoto, T., Nomoto, K., Hashimoto, M., Yanagida, S., \& Thielemann, F.-K. 1993, in Nuclei in Cosmos, ed. F. Käppeler \& K. Wisshak (Institute Physics Publ.), p. 581

Tsujimoto, T., Nomoto, K., Hashimoto, M., \& Thielemann, F.-K. 1994, ApJ, submitted

Weaver, T., \& Woosley, S.E. 1993, Phys. Rep. 227, 65

Wheeler, J.C., \& Filippenko, A.V. 1993, in this volume

Woosley, S.E., 1993, in this volume 